\title{
Labetalol Hypotensive Anesthetic Protocol paves the way to Safe Open Abdominal Myomectomy: Comparative study versus Tourniquet Hemostasis
}

'Hesham M Abo Ragab MD, ${ }^{2}$ Ahmed M Hagras MD, ${ }^{3}$ Ahmed E Salem MD, 4lnas F. Abdul Aal MD

'Department of Obstetrics \& Gynecology, Benha, Qalyobia, Egypt; Faculty of Medicine, Benha University, Egypt ${ }^{2}$ Department of Obstetrics \& Gynecology, Tanta, Gharbia, Egypt; Faculty of Medicine, Tanta University, Egypt

${ }^{3}$ Department of Anesthesia, Tanta, Gharbia, Egypt; Faculty of Medicine, Tanta University, Egypt 4Department of Anesthesia,

Cairo, Egypt, Faculty of Medicine, Cairo University, Egypt
Hesham M Abo Ragab; of Obstetrics \& Gynecology, Faculty of Medicine, Benha University, Egypt; ragabhesham2017@gmail. com

\section{Labetalol Hypotension for open Myomectomy \\ Research type: Original research.}

Funding support: There is no funding support to this research.

Disclosure: Research group has no relevant financial or nonfinancial relationships to disclose.

\begin{abstract}
Study Hypothesis: Hypotensive protocol consisted of oral labetalol pre-medication and low-dose intraoperative (IO) labetalol infusion can improve surgical outcome of open abdominal myomectomy (OAM) than IO tourniquet compression.
\end{abstract}

Study Design: Eighty-six women were randomly allocated into two equal groups: control group received normotensive anesthesia with application of uterine tourniquet for IO hemostasis and study group included patients followed the hypotensive protocol till completion of myomectomy. Surgical and anesthetic outcomes and surgeons' satisfaction were determined.

Results: Three study patients required tourniquet application for control of bleeding. In study patients, mean field visibility score was non-significantly higher, but operative time, IO blood loss and need for transfusion were significantly lower and IO urine output was significantly higher. PO hemoglobin deficit was significantly lower. Hypotensive protocol allowed significant blunting of pressor reflexes to tracheal intubation and extubation and maintained nonsingificant variations of heart rate (HR) and mean arterial pressure (MAP) measures during myomectomy. Postoperative HR and MAP measures were significantly lower in study patients compared to their baseline and to PO measures in patients of control group till 6-hr PO. Frequency of patients developed PO bleeding and hospital stay was significantly lower in study group. Surgeons' satisfaction by hypotensive anesthesia for OAM was non-significantly higher than by tourniquet hemostasis.

Conclusion: Hypotensive anesthesia using oral labetalol premedication and low-dose IO labetalol infusion significantly reduced HR and MAP till immediate PO period versus classic IO tourniquet compression. Proper hemodynamic control significantly reduced open myomectomy associated bleeding complications, improved field visibility and so allowed perfect hemostasis within significantly shorter operative time than with tourniquet control of hemostasis. 


\section{Introduction}

Uterine fibroids are common benign neoplasms with a higher prevalence ${ }^{(1)}$. Uterine fibroids can significantly impact a woman's health, fertility, and quality of life ${ }^{(2)}$. Fibroids can cause abnormal uterine bleeding, pelvic pressure manifestations, low back pain, or discovered incidentally (1). When surgery is indicated; the gold standard in uterine-sparing surgery is myomectomy ${ }^{(2)}$, but intraoperative bleeding is still a challenge ${ }^{(2)}$ as it can necessitate emergency blood transfusion if excessive or was life-threatening ${ }^{(3)}$.

Numerous medical and surgical techniques were tried to minimize potentially significant blood loss during myomectomies ${ }^{(4)}$. Preparation using gonadotropin-releasing hormone analogues for 3 -month treatment prior to myomectomy ${ }^{(5)}$ or prophylactic tranexiemic acid treatment were tried to reduce operative bleeding during abdominal myomectomy ${ }^{(6)}$. Hypotensive anesthesia allowed significant decrease of blood loss without compromising vital organ perfusion (7) and subsequently reduces transfusion requirements and minimizes allogenic transfusions risks ${ }^{(8)}$.

Labetalol is a combined $\beta$ - and $\alpha 1$-adrenoceptors antagonist ${ }^{(9)}$. Oral labetalol is readily absorbed in man ${ }^{(10)}$ with a peak plasma level was achieved after 2.5-minute and 20-120 minutes after intravenous and oral administration, respectively ${ }^{(11)}$ peak effect within 1-4 $\mathrm{hr}$ after oral intake ${ }^{(8)}$.

\section{Hypothesis}

Hypotensive protocol consisted of oral labetalol pre-medication and low-dose intraoperative (IO) labetalol infusion can improve surgical outcome of open abdominal myomectomy (OAM) than IO tourniquet compression.

\section{Objectives}

Evaluation of surgical outcome in the form of IO and postoperative (PO) bleeding, field visibility and frequency of blood transfusion in women undergoing OAM

\section{Design}

Prospective comparative study

\section{Setting}

University and Insurance hospitals, Tanta and Benha, Egypt

\section{Patients \& Methods}

After approval of the protocol of the study by the Local Ethical Committee, women required OAM and signed patients' consent were enrolled in the study. Exclusion criteria included presence of multiple myomas necessitating hysterectomy, gynecological malignancy, cardiac, renal, hepatic diseases, or history of coagulopathy. All patients underwent full history taking, general examination, complete gynecological examination, transvaginal ultrasonography (TVU) and underwent routine laboratory investigations including complete blood count. Women with hemoglobin concentration of $\leq 7 \mathrm{gm} / \mathrm{dl}$ received preoperative blood transfusion to adjusted $\mathrm{Hb}$. Conc. of $\geq 8 \mathrm{gm} / \mathrm{dl}$.

Patients who fulfilled inclusion criteria were allocated into two equal study using sealed envelopes as a method for randomization; envelops included title of the group and were prepared by an assistant who is blinded about the anesthetic procedure. Control group included patients received normotensive anesthesia with application of uterine tourniquet as a modality for bleeding control and Study group included patients received hypotensive anesthesia.

\section{Hypotensive protocol}

Baseline hemodynamic variables of study group patients were determined and patients were asked to swallow one labetalol tablet (Trandate, 200 mg tablets, Agzakhana, Egypt) two hours prior to surgery and were transferred to theater while they were maintained in supine position and preoperative heart rate (HR) and mean arterial pressure (MAP) were determined non-invasively. Labetalol infusion was prepared by clinical pharmacists to provide 0.2 $\mathrm{mg} / \mathrm{kg} / \mathrm{hr}$ infusion rate ${ }^{(12)}$. Labetalol infusion was started after induction of anesthesia and tracheal intubation, and its rate was adjusted to provide a target MAP of about $60 \mathrm{mmHg}{ }^{(13)}$ but not exceeding the recommended safe maximal dose of $300 \mathrm{mg}$ on occasion of infusion ${ }^{(14)}$.

\section{Anesthetic procedure}

Patients of control group were premedicated by midazolam $0.02 \mathrm{mg} / \mathrm{kg}$. Induction of anesthesia using propofol $(2 \mathrm{mg} / \mathrm{kg})$, fentanyl (1-2 ug/ $/ \mathrm{kg})$, and rocuronium $(0.6 \mathrm{mg} / \mathrm{kg})$, and was maintained with sevoflurane inhalation, and fentanyl and rocuronium were given according to patient's physiological reaction to surgical stimuli. Lungs 
were ventilated with $100 \% \mathrm{O} 2$ in air through semiclosed circle system for a tidal volume of 6-8 ml/ kg. Patients were continuously non-invasively monitored for MAP and HR.

\section{Surgical procedure}

Myomectomy was performed as described previously by Rock \& Jones ${ }^{(15)}$ through a Pfannenstiel incision. For patients of control group, a Foley catheter or red rubber catheter was passed through the windows made bilaterally in the broad ligament at the level of the internal cervical os around the cervix and then was tightened with a clamp to constrict uterine vessels. Then, vascular clamps were applied to the utero-ovarian ligaments ${ }^{(16)}$.

\section{Study outcome}

1. Surgical outcome included

- Operative time, intraoperative (IO) blood loss and frequency and amount of blood transfusion.

- Surgical field bleeding and subsequently its visibility was graded using 6-point scale: $0=$ no bleeding, $1=$ slight bleeding not necessitating evacuation, $2=$ slight bleeding that sometimes needed to be evacuated, $3=$ low bleeding as judged by infrequent use of suction to improve field visibility, $4=$ average bleeding, blood needed frequent evacuation and operative field is visible only right after evacuation and $5=$ high bleeding requiring continuous suction to allow field visibilty ${ }^{(17)}$.

- Failure of the applied hypotensive procedure was defined as the requirement to apply tourniquet and/or vascular clamps to control bleeding and allow field visibility.

- Amount of IO urine output.

- Hemoglobin deficit was calculated as the preoperative minus $\mathrm{PO}$ hemoglobin concentration.

- Frequency of PO complications especially hematoma formation.

- Surgeons' satisfaction by the applied hypotensive anesthesia was graded as satisfactory, good and dissatisfactory according to field visibility scoring and the requirement of tourniquet application to control bleeding.
2. Anesthetic outcome included

- The efficacy of pre-medication by oral labetalol on blunting of pressor reflexes during tracheal intubation as judged by hemodynamic changes in relation to preoperative measures.

- The efficacy of labetalol infusion to adjust intraoperative hemodynamic parameters as judged by non-invasive determinations every 10 minutes till end of myoma excision.

- The efficacy of labetalol on blunting of pressor reflexes during tracheal extubation as judged by HR and MAP changes in relation to measures at end of myoma excision.

- The efficacy of the received labetalol medications (preoperative oral and IO infusion) on postoperative (PO) course as judged by changes of HR and MAP measures at 1-, 2-, 4-, 6-, and 8-hr PO in relation to baseline measures.

\section{$\underline{\text { Statistical analysis }}$}

Sample size of $\geq 40$ patients per group was determined to be sufficient to detect a difference at the $5 \%$ significance level and give the trial $80 \%$ power as calculated according to Kraemer \& Thiemann ${ }^{(18)}$. Results were analyzed using Oneway ANOVA with post-hoc Tukey HSD Test and Chi-square test $\left(\mathrm{X}^{2}\right.$ test). Statistical analysis was conducted using the IBM SPSS (Version 23, 2015) for Windows statistical package. $\mathrm{P}$ value $<0.05$ was considered statistically significant.

\section{Results}

One-hundred and fifteen patients presented with manifestations of myoma disease were assessed for enrolment; 29 patients were excluded and 86 patients were eligible for randomization (Fig. 1). Enrolled patients were of mean age of $37.6 \pm 8$; range: and body mass index of $29.5 \pm 2.4$; range: $\mathrm{kg} / \mathrm{m} 2$ with non-significant $(\mathrm{p}>0.05)$ difference between patients of both groups. Twenty-four patients $(27.9 \%)$ required preoperative blood transfusion for correction of anemia.

All surgeries were conducted uneventfully; however, despite of the controlled hypotension, 3 patients $(7 \%)$ in study group required tourniquet application for control of bleeding that was unacceptable by the surgeon. These three 
cases were considered as failure of the applied hypotensive procedure to control IO bleeding down to a level satisfying the surgeon. Mean field visibility score was non-significantly $(p>0.05)$ higher in study patients with non-significantly $(p>0.05)$ higher frequency of patients among high scores. Operative time, amount of blood loss during surgery and need for transfusion were significantly $(p<0.05)$ lower. However, the amount of intraoperative urine output was significantly $(p<0.05)$ higher in patients of study group than controls (Table 1).

Oral labetalol induced significant $(\mathrm{p}<0.05)$ decrease of hemodynamic parameters determined preoperatively compared to baseline measures and to measures of patients of control group. In patients of control group; induction of anesthesia and tracheal intubation increased HR and MAP measures significantly $(\mathrm{p}<0.05)$ compared to baseline and preoperative measures, while in patients of study group, the difference was nonsignificant $(p>0.05)$ in comparison to preoperative measures but were significantly $(p<0.05)$ lower than baseline measures with significant $(p<0.05)$ difference versus measures of patients in control group.

During labetolol infusion (time of myoma excision), hemodynamic measures of patients in study group showed non-significant $(\mathrm{p}>0.05)$ difference and were significantly $(p<0.05)$ lower than the corresponding measures in patients of control group. On contrary, in control group, $\mathrm{HR}$ and MAP measures showed fluctuations with significant $(p<0.05)$ differences till end of myomectomy.

At time of extubation, HR and MAP were significantly $(\mathrm{p}<0.05)$ elevated in control patients, but non-significantly $(p>0.05)$ elevated in study patients compared to measures recorded at time of end of myomectomy with significantly $(p<0.05)$ higher measures in control versus study patients.

Postoperative hemodynamic measures were nonsignificant $(p>0.05)$ different compared to both baseline and preoperative measures of control patients. On the other hand, hemodynamic measures estimated after surgery in study patients were significantly $(p<0.05)$ lower than their baseline measures and to corresponding measures of controls till 6-hr PO. At 8-hr PO, hemodynamic measures were non-significantly lower on comparison to baseline measures and to that of control patients (Table 2).
Estimated hemoglobin concentration after surgery was significantly lower in all studied patients in comparison to their baseline levels with nonsignificant difference between patients of both groups. However, the deficit in hemoglobin concentration was significantly lower in patients of study versus control groups. Times till 1st ambulation and oral intake were non-significantly $(\mathrm{p}>0.05)$ longer in control versus study patients. Seventy-eight (90.7) patients passed smooth immediate PO course free of complications and were discharged after mean duration of $25 \pm 8$; range: $15-48 \mathrm{hr}$ with non-significant difference between both groups. Six patients developed PO bleeding; 5 control and one study patients with significantly $(p=0.035)$ lower frequency among study patients. Four patients developed intramural hematoma and two developed minimal pelvic collection detected on TVU. Fortunately, all cases were managed conservatively and only one control patient required blood transfusion for management of concomitant lower hemoglobin concertation. Another two patients; one in each group, developed wound infection that responded to wound cleaning, frequent dressing and extensive antibiotic therapy. These eight patients had stayed in hospital for a mean duration of $3 \pm 0.8 ; 2-4$ days. Total duration of hospitalization was significantly $(p=0.020)$ longer in control patients. Surgeons found hypotensive anesthesia for OAM was satisfactory in 33 patients, good in 7 patients and dissatisfactory in 3 patients with non-significantly $(p>0.05)$ higher satisfaction by hypotensive anesthesia than tourniquet hemostasis (Table 3).

\section{Discussion}

Open abdominal myomectomy is still the most frequently applied uterus preserving management for myoma disease especially in under-developed countries for its lower overall expenses ${ }^{(19)}$ and possibility of achieving total or near-total fibroid clearance in the majority of patients ${ }^{(20)}$. Moreover, multiple studies reported no difference in the rate of complications or recurrence after open versus laparoscopic resection ${ }^{(21,22)}$ and concerning fertility preservation versus hystroscopic resection ${ }^{(23)}$.

The current study tried to improve surgical outcome of open myomectomy through application of hypotensive anesthesia using simple cheap approach consisted of oral preparation and IO infusion of labetalol to reduce intraand postoperative bleeding which is the still 
documented (24) complication of open abdominal myomectomy.

Tourniquet application was used to achieve hemostasis in control patients; however, the amount of IO bleeding, transfusion requirement and hemoglobin deficit were significantly higher in control versus study patients. These findings indicated that the use of hypotensive anesthesia to reduce IO bleeding override the use of compression tourniquet. After infusion discontinuation, field visibility was better than after release of tourniquet, thus allowing perfect hemostasis within shorter time than after release of tourniquet and this was reflected as significantly shorter operative time in study versus control patients. This can be attributed to the long elimination half-life of labetalol that can extend for 5-8 hours ${ }^{(10)}$ thus allowing maintenance of MAP significantly lower compared to control group. Such explanation could be also applied for the reported lower frequency of postoperative bleeding in study versus control group. The reported data go in hand with that previously documented in literature concerning the impact of hypotensive anesthesia on blood loss during surgery with subsequent improvement of surgical field visibility $(25,26,27)$.

Intraoperative MAP measurements showed nonsignificant differences throughout duration of myomectomy in patients of the study groups and were significantly lower that corresponding measure in patients of control group; thus illustrating the proper hemodynamic control of labetalol infusion hemodynamic alterations induced by surgical manipulation. In line with the use of labetalol infusion for maintenance of low IO blood pressure, Jeong et al. (28) reported a significant reduction of mean arterial pressure of patients received both sevoflurane and labetalol compared to those received sevoflurane only. Moreover, Hadavi et al. (29) and El-Shmaa et al. (30) assured the effectiveness and safety of both labetalol and nitroglycerine infusions for controlled hypotension during rhinoplastic (29) and sinus endoscopic surgery (30). In support of efficacy of IO labetalol, El-Shmaa et al. (30) documented that labetalol infusion is more beneficial for providing mild hypotension with optimum operative condition, decreased surgical bleeding and less tachycardia during the surgery. Furthermore, the reported hemodynamic control of the applied protocol of hypotensive anesthesia coincided and superseded that previously reported by the authors (31) on comparison this hypotensive protocol versus placebo infusion given to control group who received neither hypotensive anesthesia nor intraoperative tourniquet.

Moreover, the beneficial effects of using oral labetalol pre-medication on hemodynamic variables evaluated preoperatively and after both induction of anesthesia and intubation, go in hand with that previously reported in literature concerning labetalol premedication and intraoperative use ${ }^{(32,33,34)}$. To our knowledge, this is the first trial using oral labetalol for preoperative preparation whenever deliberate hypotension is required. However, the obtained results supported that previously documented, regarding the use of oral $\beta$-blockers for preoperative preparation, by Apipan \& Rummasak ${ }^{(35)} \mathbf{A m r} \& \mathbf{A m i n}^{(36,37)}$ and Kim et al. ${ }^{(38)}$ who reported that premedication with oral propranolol ${ }^{(35)}$, oral atenolol ${ }^{(36,37)}$ or oral enalapril (38) before hypotensive anesthesia effectively allowed heart rate reduction with decreased amount of blood loss that allowed better quality of surgical field and reduction of amount of blood transfusion.

Estimated blood pressure measures immediately after surgery and at two hours later were significantly lower in patients received hypotensive protocol than their baseline measures and control measures determined synchronously. These data indicated that labetalol allowed proper emergence from anesthesia that was maintained postoperatively and coincided with that previously reported versus nicardipine ${ }^{(31)}$, esmolol ${ }^{(39)}$ or placebo ${ }^{(31,40)}$. Moreover, Sirivanasandha et al. ${ }^{(41)}$ documented that labetalol is a good alternative drug to control hypertensive response during emergence from anesthesia for post-craniotomy.

\section{Conclusion}

Hypotensive anesthesia protocol consisted of preoperative oral labetalol and intraoperative labetalol infusion significantly reduced hemodynamic measures with an action extended to the immediate PO period versus classic IO tourniquet compression. Proper hemodynamic control significantly reduced open myomectomy associated bleeding complications, improved field visibility and so allowed perfect hemostasis within significantly shorter operative time than with tourniquet control of bleeding. 


\section{Acknowledgments}

Authors thankClinicalPharmacistsatBenha\&Tanta University Hospitals for preparation of infusions. Also, authors acknowledge MSRA Scientific Teamwork, for Proofreading and Styling of this work (https://www.facebook.com/MSRA-2017Scientific-Organization-1826538560939267)

\section{References}

1. De La Cruz MS, Buchanan EM: Uterine Fibroids: Diagnosis and Treatment. Am Fam Physician. 2017; 95(2):100-107.

2. Flyckt R, Coyne K, Falcone T: Minimally Invasive Myomectomy. Clin Obstet Gynecol. 2017 Jan 24. doi: 10.1097/GRF.0000000000000275. [Epub ahead of print]

3. Kongnyuy EJ, Wiysonge CS: Interventions to reduce haemorrhage during myomectomy for fibroids. Cochrane Database Syst Rev. 2014; (8):CD005355.

4. Hickman LC, Kotlyar A, Shue S, Falcone T: Hemostatic Techniques for Myomectomy: An Evidence-Based Approach. J Minim Invasive Gynecol. 2016; 23(4):497-504.

5. Ferrero S, Alessandri F, Vellone VG, Venturini PL, Leone Roberti Maggiore U: Three-month treatment with ulipristal acetate prior to laparoscopic myomectomy of large uterine myomas: a retrospective study. Eur J Obstet Gynecol Reprod Biol. 2016; 205:43-7.

6. Topsoee MF, Settnes A, Ottesen B, Bergholt T: A systematic review and meta-analysis of the effect of prophylactic tranexamic acid treatment in major benign uterine surgery. Int $\mathrm{J}$ Gynaecol Obstet. 2017; 136(2):120-7.

7. Choi WS, Samman N.: Risks and benefits of deliberate hypotension in anesthesia: a systematic review. Int J Oral Maxillofac Surg. 2008; 37(8):687703.

8. Ervens J, Marks C, Hechler M, Plath T, Hansen D, Hoffmeister B. Effect of induced hypotensive anaesthesia vs isovolaemic haemodilution on blood loss and transfusion requirements in orthognathic surgery: a prospective, single-blinded, randomized, controlled clinical study. Int J Oral Maxillofac Surg. 2010; 39(12):1168-74.

9. MacCarthy EP, Bloomfield SS. Labetalol: a review of its pharmacology, pharmacokinetics, clinical uses and adverse effects. Pharmacotherapy. 1983; 3(4):193-219.
10. Jouppilla P, Kirkinen P, Keivula, Ylikorkala. Labetalol does not alter the placental and fetal blood flow or maternal prostanoids in preeclampsia. $\mathrm{Br} \mathrm{J}$ Obstet Gynaecol, 1986;93:543-7.

11. Conner CS: Labetalol: an alpha- and beta-blocker. Drug Intell Clin Pharm. 1983; 17(7-8):543-4.

12. Chauvin M, Deriaz H, Viars P: Continuous i.v. infusion of labetalol for postoperative hypertension. Haemodynamic effects and plasma kinetics. Br J Anaesth. 1987; 59(10):1250-6.

13. Goldberg ME, McNulty SE, Azad SS, Cantillo J, Torjman M, Marr AT, Huffnagle S, Seltzer JL: A comparison of labetalol and nitroprusside for inducing hypotension during major surgery. Anesth Analg. 1990 May;70(5):537-42.

14. Fahed S, Grum DF, Papadimos TJ: Labetalol infusion for refractory hypertension causing severe hypotension and bradycardia: an issue of patient safety. Patient Saf Surg. 2008; 2:13.

15. Rock JA, Jones HW. Te Linde's Operative Gynecology. 10th ed. Philadelphia, Pa: Lippincott Williams \& Wilkins; 2008.

16. DeLancey JO. A modified technique for hemostasis during myomectomy. Surg Gynecol Obstet. 1992; 174(2):153-4.

17. Fromme GA, MacKenzie RA, Gould AB, Lund BA, Offord KP: Controlled hypotension for orthognatic surgery. Anesth Analg., 1986; 65: 683-6.

18. Kraemer HC, Theimann S: How many subjects? Statistical power analysis in research; 1987, Newbury Park, CA: Sage.

19. Chang CC: A comparison of the costs of laparoscopic myomectomy and open myomectomy at a teaching hospital in southern Taiwan. Taiwan J Obstet Gynecol. 2013; 52(2):227-32.

20. Wijesekera NT, Mauri G, Gupta S, Belli AM, Manyonda IT: MR imaging evaluation of fibroid clearance following open myomectomy for massive/multiple symptomatic fibroids. Arch Gynecol Obstet. 2012 Nov;286(5):1165-71.

21. Chang CC, Chen W: A comparison of surgical outcomes between laparoscopic and open myomectomy in Southern Taiwan. Int J Gynaecol Obstet. 2012; 119(2):189-93.

22. Iavazzo C, Mamais I, Gkegkes ID: Robotic assisted vs laparoscopic and/or open myomectomy: systematic review and meta-analysis of the clinical evidence. Arch Gynecol Obstet. 2016;294(1):5-17.

23. Capmas P, Voulgaropoulos A, Legendre G, 
Pourcelot AG, Fernandez H: Hysteroscopic resection of type 3 myoma: a new challenge? Eur J Obstet Gynecol Reprod Biol. 2016; 205:165-9.

24. Conforti A, Mollo A, Alviggi C, Tsimpanakos I, Strina I, Magos A, et al.: Techniques to reduce blood loss during open myomectomy: a qualitative review of literature. Eur J Obstet Gynecol Reprod Biol. 2015; 192:90-5.

25. Prasant MC, Kar S, Rastogi S, Hada P, Ali FM, Mudhol A: Comparative Study of Blood Loss, Quality of Surgical Field and Duration of Surgery in Maxillofacial Cases with and without Hypotensive Anesthesia. J Int Oral Health. 2014; 6(6):18-21.

26. Nazir O, Wani MA, Ali N, Sharma T, Khatuja A, Misra R, et al.: Use of Dexmedetomidine and Esmolol for Hypotension in Lumbar Spine Surgery. Trauma Mon. 2016; 21(3):e22078.

27. Lin S, McKenna SJ, Yao CF, Chen YR, Chen C: Effects of Hypotensive Anesthesia on Reducing Intraoperative Blood Loss, Duration of Operation, and Quality of Surgical Field During Orthognathic Surgery: A Systematic Review and Meta-Analysis of Randomized Controlled Trials. J Oral Maxillofac Surg. 2017; 75(1):73-86.

28. Hadavi MR, Zarei Y, Tarogh S: Comparison of effects of labetalol and nitroglycerine on intraoperative blood loss and surgical field quality in rhinoplasty surgery. World J Plast Surg. 2015; 4(1):60-5.

29. Jeong J, Portnof JE, Kalayeh M, Hardigan P: Hypotensive anesthesia: Comparing the effects of different drug combinations on mean arterial pressure, estimated blood loss, and surgery time in orthognathic surgery. J Craniomaxillofac Surg. 2016; 44(7):854-8.

30. El-Shmaa NS, Ezz HAA, Younes A: The efficacy of Labetalol versus Nitroglycerin for induction of controlled hypotension during sinus endoscopic surgery. A prospective, double-blind and randomized study. J Clin Anesth. 2017; 39:154-8.

31. Salem AE, Hagras AM, Abo-Ragab HM: Labetalol Hypotensive Anesthetic Protocol Paves the Way to Safe Open Abdominal Myomectomy. J Anest \& Inten Care Med. 2017: 3(2): 555606.

32. Ryu JH, Apfel CC, Whelan R, Jeon YT, Hwang JW, Do SH, et al.: Comparative prophylactic and therapeutic effects of intravenous labetalol 0.4 $\mathrm{mg} / \mathrm{kg}$ and nicardipine $20 \mu \mathrm{g} / \mathrm{kg}$ on hypertensive responses to endotracheal intubation in patients undergoing elective surgeries with general anesthesia: a prospective, randomized, doubleblind study. Clin Ther. 2012; 34(3):593-604.
33. Meftahuzzaman SM, Islam MM, Ireen ST, Islam MR, Kabir H, Rashid H, et al.: Comparison of efficacy of labetalol and fentanyl for attenuating reflex responses to laryngoscopy and intubation. Mymensingh Med J. 2014; 23(2):242.-8.

34. El-Shmaa NS, El-Baradey GF: The efficacy of labetalol vs dexmedetomidine for attenuation of hemodynamic stress response to laryngoscopy and endotracheal intubation. J Clin Anesth. 2016; $31: 267-73$.

35. Apipan B, Rummasak D: Efficacy and safety of oral propranolol premedication to reduce reflex tachycardia during hypotensive anesthesia with sodium nitroprusside in orthognathic surgery: a double-blind randomized clinical trial. J Oral Maxillofac Surg. 2010; 68(1):120-4.

36. Amr YM, Amin SM: Effects of preoperative oral beta blocker versus intraoperative nitroprusside or esmolol on quality of surgical field during tympanoplasty. J Clin Anesth. 2011; 23(7):544-8.

37. Amr YM, Amin SM: Effects of preoperative $\beta$-blocker on blood loss and blood transfusion during spinal surgeries with sodium nitroprussidecontrolled hypotension. Saudi J Anaesth. 2012; 6(3):263-7.

38. Kim NY, Yoo YC, Chun DH, Lee HM, Jung YS, Bai SJ: The Effects of Oral Atenolol or Enalapril Premedication on Blood Loss and Hypotensive Anesthesia in Orthognathic Surgery. Yonsei Med J. 2015; 56(4):1114-21.

39. Mashour GA, Sharifpour M, Freundlich RE, Tremper KK, Shanks A, Nallamothu BK, et al.: Perioperative metoprolol and risk of stroke after noncardiac surgery. Anesthesiology. 2013; $119(6): 1340-6$.

40. Sanath Kumar SB, Date R, Woodhouse N, ElShafie O, Nollain K: Successful Management of Phaeochromocytoma using Preoperative Oral Labetalol and Intraoperative Magnesium Sulphate: Report of four cases. Sultan Qaboos Univ Med J. 2014; 14(2):e236-40.

41. Sirivanasandha B, Sakaew A, Sutthivaiyakit K, RaksamaniK, Waitayawinyu P, Rushatamukayanunt $P$, et al: An Equivalence Trial Comparing Labetalol and Diltiazem in Controlling Emergence Hypertension after Supratentorial Tumor Surgery. J Med Assoc Thai. 2015; 98(11):1104-11. 